\begin{tabular}{|c|l|}
\hline Title & A Generalization of the Moritz Mumford Classes to Extended Mapping Class Groups for Surfaces \\
\hline Author(s) & Kawazumi, N. \\
\hline Citation & Hokkaido University Preprint Series in Mathematics, 292, 1-11 \\
\hline Issue Date & 1995-4-1 \\
\hline DOI & 10.14943/83439 \\
\hline Doc URL & http://hdl.handle.net/2115/69043 \\
\hline Type & bulletin (article) \\
\hline File Information & pre292.pdf \\
\hline
\end{tabular}

Instructions for use 


\title{
A GENERALIZATION OF THE MORITA-MUMFORD CLASSES TO EXTENDED MAPPING CLASS GROUPS FOR SURFACES
}

\author{
Nariya Kawazumi
}

Series $\sharp 292$. April 1995 


\section{HOKKAIDO UNIVERSITY PREPRINT SERIES IN MATHEMATICS}

$\sharp 269$ A. Arai, Operator-theoretical analysis of representation of a supersymmetry algebra in Hilbert space, 12 pages. 1994.

$\sharp 270$ A. Arai, Gauge theory on a non-simply-connected domain and representations of canonical commutation relations, 18 pages. 1994 .

$\sharp 271$ S. Jimbo, Y. Morita and J. Zhai, Ginzburg landau equation and stable steady state solutions in a non-trivial domain, 17 pages. 1994.

$\sharp 272$ S. Izumiya, A. Takiyama, A time-like surface in Minkowski 3-space which contains light-like lines, 7 pages. 1994.

$\sharp 273$ K. Tsutaya, Global existence of small amplitude solutions for the Klein-Gordon-Zakharov equations, 11 pages. 1994.

$\sharp 274$ H. Kubo, On the critical decay and power for semilinear wave equations in odd space dimensions, 22 pages. 1994.

$\sharp 275$ N. Terai, T. Hibi, Alexander duality theorem and second Betti numbers of Stanley-Reisner rings, 2 pages. 1995.

$\sharp 276$ N. Terai, T. Hibi, Stanley-Reisner rings whose Betti numbers are independent of the base field, 12 pages. 1995.

$\sharp 277$ N. Terai, T. Hibi, Computation of Betti numbers of monomial ideals associated with cyclic polytopes, 11 pages. 1995.

$\sharp 278$ N. Terai, T. Hibi, Computation of Betti numbers of monomial ideals associated with stacked polytopes, 8 pages. 1995.

$\sharp 279$ N. Terai, T. Hibi, Finite free resolutions and 1-skeletons of simplicial $(d-1)$-spheres, 3 pages. 1995.

$\sharp 280$ N. Terai, T. Hibi, Monomial ideals and minimal non-faces of Cohen-Macaulay complexes, 6 pages. 1995.

\#281 A. Arai, N. Tominaga, Analysis of a family of strongly commuting self-adjoint operators with applications to perturbed d'Alembertians and the external field problem in quantum field theory, 44 pages. 1995.

$\sharp 282$ T. Mikami, Asymptotic behavior of the first exit time of randomly perturbed dynamical systems with a repulsive equilibrium point, 29 pages. 1995.

$\sharp 283$ K. Iwata, J. Schäfer, Markov property and cokernels of local operators, 17 pages. 1995.

\#284 T. Nakazi, M. Yamada, Riesz's Functions In Weighted Hardy And Bergman Spaces, 20 pages. 1995.

$\sharp 285$ K. Hidano, K. Tsutaya, Scattering theory for nonlinear wave equations in the invariant Sobolev space, 32 pages. 1995.

$\sharp 286$ A. Arai, Strong coupling limit of the zero-energy-state density of the Dirac-Weyl operator with a singular vector potential, 8 pages. 1995 .

$\sharp 287$ T. Nakazi, Factorizations of outer functions and extremal problems, 15 pages. 1995.

$\sharp 288$ A. Kishimoto, The Rohlin property for automorphisms of UHF algebras, 15 pages. 1995.

$\sharp 289$ K. Goto, A. Yamaguchi and I. Tsuda, Nine-bit states cellular automata are capable of simulating the pattern dynamics of coupled map lattice, 24 pages. 1995.

\#290 Y. Giga, Interior derivative blow-up for quasilinear parabolic equations, 16 pages. 1995.

\#291 F. Hiroshima, Functional Integral Representation of a Model in QED, 48 pages. 1995. 


\title{
A GENERALIZATION OF THE MORITA-MUMFORD CLASSES TO EXTENDED MAPPING CLASS GROUPS FOR SURFACES
}

\author{
NARIYA KAWAZUMI \\ Department of Mathematics, Faculty of Sciences, \\ Hokkaido University, Sapporo, 060 Japan
}

\begin{abstract}
Let $\Sigma_{g, 1}$ be an orientable compact surface of genus $g$ with 1 boundary component, and $\Gamma_{g, 1}$ the mapping class group of $\Sigma_{g, 1}$. We define a bigraded series of cohomology classes $m_{i, j} \in H^{2 i+j-2}\left(\Gamma_{g, 1} ; \bigwedge^{j} H_{1}\left(\Sigma_{g, 1} ; \mathbb{Z}\right)\right), 2 i+j-2 \geq 1, i, j \geq 0$. When $j=0$, the class $m_{i+1,0}$ is the $i$-th Morita-Mumford class [Mo][Mu]. It is proved that $H^{r}\left(\Gamma_{g, 1} ; \bigwedge^{s} H_{1}\left(\Sigma_{g, 1} ; \mathbb{Q}\right)\right)$ is generated by $m_{i, j}$ 's for the case $r+s=2$ and the case $g \geq 5$ and $(r, s)=(1,3)$. Especially the Johnson homomorphism extended to the whole mapping class group by Morita [Mo3] has an implicit representation by the classes $m_{0,3}$ and $m_{0,2} m_{1,1}$ over $\mathbb{Q}$.
\end{abstract}

\section{INTRODUCTION}

Let $g \geq 2, r, n \geq 0$ be integers. Let $\Sigma_{g, r}^{n}$ denote a 2-dimensional compact oriented $C^{\infty}$ manifold (i.e., compact oriented surface) of genus $g$ with $r$ boundary components and (ordered) $n$ punctures. The group of path-components $\pi_{0}\left(\operatorname{Diff}^{+}\left(\Sigma_{g, r}^{n}\right)\right)$ is denoted by $\Gamma_{g, r}^{n}\left(\right.$ or $\left.\mathcal{M}_{g, r}^{n}\right)$ and called the mapping class group of genus $g$ with $r$ boundary components and (ordered) $n$ punctures. Here $\operatorname{Diff}^{+}\left(\Sigma_{g, r}^{n}\right)$ denotes the topological group (endowed with $C^{\infty}$ topology) consisting of all orientation preserving diffeomorphisms of $\Sigma_{g, r}^{n}$ which fix the boundary components and the punctures pointwise. When $n=0$, we drop the indices: $\Sigma_{g, r}=\Sigma_{g, r}^{0}, \Gamma_{g, r}=\Gamma_{g, r}^{0}$ and similarly $\Sigma_{g}=\Sigma_{g, 0}^{0}, \Gamma_{g}=\Gamma_{g, 0}^{0}$. Throughout this paper we denote by $H_{1}\left(\Sigma_{g, r}^{n}\right)$ the first integral singular homology of the space $\Sigma_{g, r}^{n}$, on which the group $\Gamma_{g, s}^{m}$ act in an obvious way provided that $s \geq r$ and $m \geq n$.

By the extended mapping class group we mean the semi-direct product

$$
\widetilde{\Gamma_{g, r}^{n}}:=H_{1}\left(\Sigma_{g, 1}\right) \rtimes \Gamma_{g, r}^{n} .
$$

The purpose of the present paper is to define a bigraded series $\widetilde{m_{i, j}}$ of cohomology classes of the extended group $\widetilde{\Gamma_{g, 1}}$, which is a generalization of the Morita-Mumford cohomology classes of the group $\Gamma_{g}$, and to investigate the ones of lower degree.

In $\S 1$ we prepare a theory of cohomology of pairs of groups, which is essential to the construction of the classes in the succeeding two sections. The $E_{2}$-term of the

1991 Mathematical Subject Classification. Primary 57R20. Secondary 14H15, 20J05, 57R32, 20F36. 
Lyndon-Hochschild-Serre spectral sequence of the group $\widetilde{\Gamma_{g, 1}}$ with respect to the normal subgroup $H_{1}\left(\Sigma_{g, 1}\right)$ is given by

$$
E_{2}^{p, q}=H^{p}\left(\Gamma_{g, 1} ; \bigwedge^{q} H^{1}\left(\Sigma_{g, 1}\right)\right)
$$

So the classes $\widetilde{m_{i, j}}$ induce cohomology classes $m_{i, j}$ of the group $\Gamma_{g, 1}$ with values in $\wedge^{*} H^{1}\left(\Sigma_{g, 1}\right)$. When $j=0$, the class $m_{i+1,0}$ is the $i$-th Morita-Mumford class [Mo][Mu]. In $\S 4$, in order to see the non-triviality, we evaluate the classes $m_{0,2}$, $m_{1,1}$ and $m_{0,3}$ and prove that $H^{r}\left(\Gamma_{g, 1} ; \bigwedge^{s} H_{1}\left(\Sigma_{g, 1} ; \mathbb{Q}\right)\right)$ is generated by $m_{i, j}$ 's for the case $r+s=2$ (Proposition 4.1, Theorem 4.2, Corollary 4.6) and the case $g \geq 5$ and $(r, s)=(1,3)$ (Theorem 4.5). Especially the Johnson homomorphism extended to the whole mapping class group by Morita [Mo3] has an implicit representation by the classes $m_{0,3}$ and $m_{0,2} m_{1,1}$ over $\mathbb{Q}$.

The author would like to express his gratitude to Prof. S. Morita and Prof. A. Kohno for helpful discussions.

Contents.

$\S 1$. Cohomology of Pairs of Groups.

§2. Mapping Class Groups.

$\S 3$. Construction of Cohomology Classes.

$\S 4$. Evaluations.

\section{Cohomology of Pairs of Groups.}

In this section we define cohomology groups $H^{*}(G, H: M)$ of a pair of groups $(G, H)$ in the most naive sense. Denote by $C^{*}(G ; M)$ the normalized cochain complex of a group $G$ with values in a $G$-module $M$.

Let $G$ be a group, $H$ a subgroup of $G$, and $M$ a $G$-module. We denote by $H^{*}(G, H ; M)$ the cohomology group of the kernel of the restriction map

$$
\text { res : } C^{*}(G ; M) \rightarrow C^{*}(H ; M)
$$

and call it the cohomology group of the pair of groups $(G, H)$ with values in the $G$-module $M$. Since the restriction map res is surjective in the cochain level, we have a cohomology exact sequence

$$
\cdots \rightarrow H^{q-1}(H ; M) \rightarrow H^{q}(G, H ; M) \rightarrow H^{q}(G ; M) \rightarrow H^{q}(H ; M) \rightarrow \cdots
$$

In a natural way the cup product

$$
\cup: H^{*}\left(G ; M^{\prime}\right) \otimes H^{*}\left(G, H ; M^{\prime \prime}\right) \rightarrow H^{*}\left(G, H ; M^{\prime} \otimes M^{\prime \prime}\right)
$$

is defined.

Let $K \triangleleft G$ be a normal subgroup satisfying the condition

$$
H K=G
$$

Then we have the following Lyndon-Hochshild-Serre (LHS) spectral sequence [HS]. 
Proposition 1.3. There is a spectral sequence converging to $H^{*}(G, H ; M)$ whose $E_{2}$ term is given by

$$
E_{2}^{p, q}=H^{p}\left(G / K ; H^{q}(K, K \cap H ; M)\right)
$$

It should be remarked how the quotient group $G / K$ acts on the cohomology group $H^{*}(K, K \cap H ; M)$. Since $K$ is a normal subgroup of $G$, the group $H$ acts on the normalized complex $C^{*}(K, K \cap H ; M)$ by

$$
(h \cdot c)\left(x_{1}, \ldots, x_{n}\right):=h\left(c\left(h^{-1} x_{1} h, \ldots, h^{-1} x_{n} h\right)\right)
$$

where $h \in H, c \in C^{n}(K, K \cap H ; M)$ and $x_{1}, \ldots, x_{n} \in K$. For any element $h \in K \cap H$ consider a homotopy map

$$
\Phi=\Phi_{h}: C^{n}(K, K \cap H ; M) \rightarrow C^{n-1}(K, K \cap H ; M)
$$

given by

$$
\left(\Phi_{h} c\right)\left(x_{1}, \ldots, x_{n-1}\right):=\sum_{j=0}^{n-1}(-1)^{j} c\left(x_{1}, \ldots, x_{j}, h, h^{-1} x_{j+1} h, \ldots, h^{-1} x_{n-1} h\right)
$$

This map is well-defined and satisfies a homotopy equation

$$
\left(d \Phi_{h}+\Phi_{h} d\right) c=h \cdot c-c \quad\left(\forall c \in C^{*}(K, K \cap H ; M)\right)
$$

Hence the subgroup $K \cap H$ acts on the cohomology group $H^{*}(K, K \cap H ; M)$ trivially. From the condition (1.2) and the Second Isomorphism Theorem we have a natural isomorphism

$$
G / K=H / K \cap H
$$

Thus the quotient group $G / K$ acts on the cohomology group $H^{*}(K, K \cap H ; M)$.

Let $M, M_{1}$ and $M_{2}$ be $G / K$-modules. Suppose

$$
H^{q}(K, K \cap H ; \mathbb{Z})= \begin{cases}\mathbb{Z}, & \text { if } q=n \\ 0, & \text { if } q>n\end{cases}
$$

Then the spectral sequence (1.3) induces a homomorphism

$$
\pi_{!}: H^{p}(G, H ; M) \rightarrow H^{p-n}(G / K ; M)
$$

which is called the Gysin map or the fiber integral. As usual we have

$$
\pi_{!}\left(u \cup \pi^{*} v\right)=(\pi ! u) \cup v \in H^{p+q-n}\left(G / K ; M_{1} \otimes M_{2}\right)
$$

for $u \in H^{p}\left(G, H ; M_{1}\right)$ and $v \in H^{q}\left(G / K ; M_{2}\right)$. 


\section{Mapping Class Groups.}

From now on we consider mainly the mapping class groups $\Gamma_{g, 1}$ and $\Gamma_{g, 1}^{1}$. First we remark that the surface $\Sigma_{g, 1}^{1}$ is obtained by glueing the surfaces $\Sigma_{g, 1}$ and $\Sigma_{0,2}^{1}$ along the boundaries. So the diffeomorphism of $\Sigma_{g, 1}$ is naturally extended to that of $\Sigma_{g, 1}^{1}$. The infinite cyclic group $\mathbb{Z}$ acts on the surface $\Sigma_{0,2}^{1}$ by rotating the puncture and fixing the boundaries pointwise. Similarly this action is extended to that on $\Sigma_{g, 1}^{1}$ in a natural way. Thus we obtain a natural homomorphism $\Gamma_{g, 1} \times \mathbb{Z} \rightarrow \Gamma_{g, 1}^{1}$, which is injective (see [I] §5). In the sequal we regard the group $\Gamma_{g, 1} \times \mathbb{Z}$ as a subgroup of $\Gamma_{g, 1}^{1}$ through the injection. Especially we may consider the cohomology group $H^{*}\left(\Gamma_{g, 1}^{1}, \Gamma_{g, 1} \times \mathbb{Z} ; M\right)$ for an arbitrary $\Gamma_{g, 1}^{1}$-module $M$. By forgetting the puncture we obtain an extension

$$
1 \rightarrow \pi_{1}\left(\Sigma_{g, 1}\right) \rightarrow \Gamma_{g, 1}^{1} \stackrel{\pi}{\rightarrow} \Gamma_{g, 1} \rightarrow 1 .
$$

Next we prepare a cycle induced by the "fiber" $\pi_{1}\left(\Sigma_{g, 1}\right)$. Choose a usual symplectic generator system of the fundamental group $\pi_{1}\left(\Sigma_{g, 1}\right)$ :

$$
a_{1}, a_{2}, \ldots, a_{g}, b_{1}, b_{2}, \ldots, b_{g} \text {. }
$$

The loop on the boundary induces an element of $\pi_{1}\left(\Sigma_{g, 1}\right)$

$$
w:=\prod_{i=1}^{g}\left[a_{i} b_{i}\right], \quad\left[a_{i}, b_{i}\right]=a_{i} b_{i} a_{i}^{-1} b_{i}^{-1} \text {. }
$$

We identify the group $\mathbb{Z}$ with the subgroup generated by $w$ in $\pi_{1}\left(\Sigma_{g, 1}\right)$, and consider the cohomology group of the pair $H^{*}\left(\pi_{1}\left(\Sigma_{g, 1}\right), \mathbb{Z}\right)$.

Following Meyer [Me], we construct a normalized bar 2-chain $\left[\Sigma_{g, 1}, \partial\right]$ as follows. For $1 \leq j \leq 4 g$ let $w_{j}=a_{i}{ }^{ \pm 1}, b_{i}{ }^{ \pm 1}$ be the $j$-th generator in the element $w$, and $\widetilde{w_{j}}:=w_{1} w_{2} \cdots w_{j}=a_{1} b_{1} \cdots w_{j}$. Let $\widetilde{w_{0}}=1$. We define

$$
\left[\Sigma_{g, 1}, \partial\right]:=\sum_{j=1}^{4 g}\left[\widetilde{w_{j-1}} \mid w_{j}\right]-\sum_{i=1}^{g}\left(\left[a_{i} \mid a_{i}^{-1}\right]+\left[b_{i} \mid b_{i}^{-1}\right]\right) \in C_{2}\left(\pi_{1}\left(\Sigma_{g, 1}\right)\right)
$$

Lemma 2.3. For any trivial $\pi_{1}\left(\Sigma_{g, 1}\right)$-module $M$, we have

$$
H^{*}\left(\pi_{1}\left(\Sigma_{g, 1}\right), \mathbb{Z} ; M\right)= \begin{cases}H \otimes M, & \text { if } *=1, \\ M, & \text { if } *=2, \\ 0, & \text { otherwise, }\end{cases}
$$

where $H=H_{1}\left(\Sigma_{g, 1} ; \mathbb{Z}\right) \cong \mathbb{Z}^{2 g}$. The evaluation

$$
<\cdot\left[\Sigma_{g, 1}, \partial\right]>: H^{2}\left(\pi_{1}\left(\Sigma_{g, 1}\right), \mathbb{Z} ; M\right) \rightarrow M
$$

is a well-defined isomorphism.

The first half of the lemma follows form the exact sequence (1.1), and the second from straightforward calculations. 
Now let $M$ be a $\Gamma_{g, 1}$-module. The condition (1.2) is satisfied for our case $G=$ $\Gamma_{g, 1}^{1}, H=\Gamma_{g, 1} \times \mathbb{Z}$ and $K=\pi_{1}\left(\Sigma_{g, 1}\right)$. It follws from Proposition 1.3 there exists a spectral sequence converging to

$$
H^{*}\left(\Gamma_{g, 1}^{1}, \Gamma_{g, 1} \times \mathbb{Z} ; M\right)
$$

whose $E_{2}$ term is given by

$$
H^{p}\left(\Gamma_{g, 1} ; H^{q}\left(\pi_{1}\left(\Sigma_{g, 1}\right), \mathbb{Z} ; M\right)\right)= \begin{cases}H^{p}\left(\Gamma_{g, 1} ; H \otimes M\right), & \text { if } *=1, \\ H^{p}\left(\Gamma_{g, 1} ; M\right), & \text { if } *=2, \\ 0, & \text { otherwise. }\end{cases}
$$

Hence it induces a Gysin exact sequence

$$
\begin{aligned}
\cdots \rightarrow H^{q-1}\left(\Gamma_{g, 1} ; M\right) \rightarrow H^{q+1} & \left(\Gamma_{g, 1} ; H \otimes M\right) \\
& \rightarrow H^{q+2}\left(\Gamma_{g, 1}^{1}, \partial \Gamma_{g, 1}^{1} ; M\right) \stackrel{\pi_{3}}{\rightarrow} H^{q}\left(\Gamma_{g, 1} ; M\right) \rightarrow \cdots
\end{aligned}
$$

Here the homomorphism $\pi_{!}$is the fiber integral introduced in (1.5).

The Gysin sequence splits as follows. The identity map $1_{\mathbb{Z}}: \mathbb{Z} \rightarrow \mathbb{Z}$ generates the cohomology group $H^{1}(\mathbb{Z}) \cong \mathbb{Z}$. Regard $1_{\mathbb{Z}}$ as an element of $H^{1}\left(\Gamma_{g, 1} \times \mathbb{Z}\right)$ through the natural projection $\Gamma_{g, 1} \times \mathbb{Z} \rightarrow \mathbb{Z}$ and denote by $\theta$ the image of $1_{\mathbb{Z}}$ under the connecting homomorphism $\delta^{*}$ :

$$
\theta:=\delta^{*}\left(1_{\mathbb{Z}}\right) \in H^{2}\left(\Gamma_{g, 1}^{1}, \partial \Gamma_{g, 1}^{1} ; \mathbb{Z}\right) .
$$

Since $<\theta,\left[\Sigma_{g, 1}, \partial\right]>=-1$, we have

$$
\pi_{!} \theta=-1 \in H^{0}\left(\Gamma_{g, 1} ; \mathbb{Z}\right) .
$$

Thus, from the property (1.6) of the fiber integral $\pi_{!}$, the sequence splits. Consequently we have

Proposition 2.5. For any $\Gamma_{g, 1}-$ module $M$, we have an exact sequence

$$
0 \rightarrow H^{q+1}\left(\Gamma_{g, 1} ; H \otimes M\right) \rightarrow H^{q+2}\left(\Gamma_{g, 1}^{1}, \partial \Gamma_{g, 1}^{1} ; M\right) \stackrel{\pi_{!}}{\rightarrow} H^{q}\left(\Gamma_{g, 1} ; M\right) \rightarrow 0,
$$

which splits as follows:

$$
H^{q+2}\left(\Gamma_{g, 1}^{1}, \partial \Gamma_{g, 1}^{1} ; M\right)=H^{q+1}\left(\Gamma_{g, 1} ; H \otimes M\right) \oplus \theta \cup H^{q}\left(\Gamma_{g, 1} ; M\right) .
$$

On the other hand, taking the semi-direct product of the extension (2.1) and the $\Gamma_{g, 1}$-module $H_{1}\left(\Sigma_{g, 1} ; \mathbb{Z}\right)$, we have an extension of groups

$$
1 \rightarrow \pi_{1}\left(\Sigma_{g, 1}\right) \rightarrow \widetilde{\Gamma_{g, 1}^{1}} \stackrel{\widetilde{\pi}}{\rightarrow} \widetilde{\Gamma_{g, 1}^{1}} \rightarrow 1 .
$$

In a similar way to the fiber integral $\pi_{\text {! }}$ we obtain the fiber integral

$$
\tilde{\pi}_{!}: H^{q}\left(\widetilde{\Gamma_{g, 1}^{1}}, \widetilde{\Gamma_{g, 1}} \times \mathbb{Z} ; \mathbb{Z}\right) \rightarrow H^{q-2}\left(\widetilde{\Gamma_{g, 1}} ; \mathbb{Z}\right) .
$$




\section{Construction of Cohomology Classes.}

For the rest we often abbreviate

$$
H:=H_{1}\left(\Sigma_{g, 1} ; \mathbb{Z}\right)=H^{1}\left(\Sigma_{g, 1} ; \mathbb{Z}\right) .
$$

The isomorphism on the right-hand side is the Poincare duality, which is $\Gamma_{g, 1^{-}}$ equivariant. We remark this $H$ plays a different role in the sequal from the subgroup $H$ in the preceeding sections.

The surface $\Sigma_{g, 1}^{1}$ may be regarded as a subspace of $\Sigma_{g, 1}$ in an obvious way, so one obtains a natural homology exact sequence

$$
0 \rightarrow H_{2}\left(\Sigma_{g, 1}, \Sigma_{g, 1}^{1}\right)=\mathbb{Z} \stackrel{\iota}{\rightarrow} H_{1}\left(\Sigma_{g, 1}^{1}\right) \rightarrow H_{1}\left(\Sigma_{g, 1}\right)=H \rightarrow 0,
$$

which induces a central extension

$$
0 \rightarrow \mathbb{Z} \rightarrow H_{1}\left(\Sigma_{g, 1}^{1}\right) \rtimes \Gamma_{g, 1}^{1} \rightarrow \widetilde{\Gamma_{g, 1}^{1}} \rightarrow 0 .
$$

Let

$$
\widetilde{\omega} \in H^{2}\left(\widetilde{\Gamma_{g, 1}^{1}} ; \mathbb{Z}\right)
$$

denote the 2-dimensional cohomology class defined by the central extension (3.2). In order to investigate the class $\tilde{\omega}$ geometrically, we choose a simple curve $l$ on $\Sigma_{g, 1}^{1}$ connecting the puncture to a point on the boundary. Define a 2 -chain $\widetilde{\omega_{l}} \in C^{2}\left(\widetilde{\Gamma_{g, 1}^{1}}\right.$; $\mathbb{Z})$ by

$$
\widetilde{\omega_{l}}\left(u_{1} \gamma_{1}, u_{2} \gamma_{2}\right):=\gamma_{1}\left(\gamma_{2} l-l\right) \cdot u_{1}, \quad u_{1}, u_{2} \in H, \gamma_{1}, \gamma_{2} \in \Gamma_{g, 1}^{1},
$$

where we remark $\gamma_{2} l-l$ can be regarded as a closed curve on $\Sigma_{g, 1}$.

Lemma 3.4. The 2-cochain $\widetilde{\omega_{l}}$ is a cocycle and its cohomology class is equal to $\widetilde{\omega}$ :

$$
\widetilde{\omega}=\left[\widetilde{\omega_{l}}\right] \in H^{2}\left(\widetilde{\Gamma_{g, 1}^{1}} ; \mathbb{Z}\right) .
$$

Proof. Since the intersection number $l \cdot \iota(1)$ is equal to 1 , the curve $l$ induces a splitting homomorphism $\sigma=\sigma_{l}: H_{1}\left(\Sigma_{g, 1}\right) \rightarrow H_{1}\left(\Sigma_{g, 1}^{1}\right)$ of the exact sequence (3.1). By definition the 2-cocycle induced by the splitting homomorphism $\sigma_{l}$ is given by

$$
\begin{aligned}
& \iota^{-1}\left(\sigma\left(u_{1}\right) \gamma_{1} \sigma\left(u_{2}\right) \gamma_{2}\left(\sigma\left(u_{1} \gamma_{1}\left(u_{2}\right)\right) \gamma_{1} \gamma_{2}\right)^{-1}\right)=\iota^{-1}\left(\gamma_{1}\left(\sigma\left(u_{2}\right)\right)-\sigma\left(\gamma_{1}\left(u_{2}\right)\right)\right) \\
= & l \cdot \gamma_{1}\left(\sigma\left(u_{2}\right)\right)-l \cdot \sigma\left(\gamma_{1}\left(u_{2}\right)\right)=l \cdot \gamma_{1}\left(\sigma\left(u_{2}\right)\right)=\left(\gamma_{1}^{-1}(l)-l\right) \cdot \sigma\left(u_{2}\right) \\
= & \left(\gamma_{1}{ }^{-1}(l)-l\right) \cdot u_{2} .
\end{aligned}
$$

Here we use the fact $l \cdot \sigma\left(u_{2}\right)=0$.

On the other hand give $c \in C^{1}\left(\widetilde{\Gamma_{g, 1}^{1}} ; \mathbb{Z}\right)$ by

$$
c(u \gamma):=l \cdot \gamma \sigma\left(\gamma^{-1}(u)\right), \quad u \in H, \gamma \in \Gamma_{g, 1}^{1},
$$


then we have

$$
d c\left(u_{1} \gamma_{1}, u_{2} \gamma_{2}\right)=\widetilde{\omega}_{l}\left(u_{1} \gamma_{1}, u_{2} \gamma_{2}\right)-\left(\gamma_{1}^{-1}(l)-l\right) \cdot u_{2}
$$

Thus $d \widetilde{\omega}_{l}=0$ and the cohomology class of $\widetilde{\omega}_{l}$ is equal to $\widetilde{\omega}$.

Next consider the 1-chain $\omega_{l} \in C^{1}\left(\Gamma_{g, 1}^{1} ; H\right)$ defined by

$$
\omega_{l}(\gamma)=\gamma l-l \in H, \quad \gamma \in \Gamma_{g, 1}^{1} .
$$

Recall $H^{1}\left(\Gamma_{g, 1}^{1} ; H\right)$ is the $E_{2}^{1,1}$-term of the LHS spectral sequence of $\widetilde{\Gamma_{g, 1}}$ with respect to the normal subgroup $H$. Since we have

$$
\widetilde{\omega_{l}}\left(u_{1}, u_{2} \gamma_{2}\right)=\omega_{l}\left(\gamma_{2}\right) \cdot u_{1}
$$

for $\forall u_{1}, u_{2} \in H$ and $\gamma_{2} \in \Gamma_{g, 1}^{1}$, the cochain $\omega_{l}$ is a 1-cocycle and its cohomology class

$$
\omega:=\left[\omega_{l}\right] \in H^{1}\left(\Gamma_{g, 1}^{1} ; H\right)
$$

is equal to that induced by $\widetilde{\omega} \in H^{2}\left(\widetilde{\Gamma_{g, 1}^{1}} ; \mathbb{Z}\right)$, and so the class is independent of the choice of $l$. We remark that

$$
\omega_{l}(\gamma)=-\gamma \in H, \quad \forall \gamma \in \pi_{1}\left(\Sigma_{g, 1}\right) .
$$

Now we can define the cohomology classes $\widetilde{m_{i, j}}$ and $m_{i, j}$. Consider two extensions of groups

$$
\begin{aligned}
& 1 \rightarrow \pi_{1}\left(\Sigma_{g, 1}\right) \rightarrow \Gamma_{g, 1}^{1} \stackrel{\pi}{\rightarrow} \Gamma_{g, 1}^{1} \rightarrow 1 \\
& 1 \rightarrow \pi_{1}\left(\Sigma_{g, 1}\right) \rightarrow \widetilde{\Gamma_{g, 1}^{1}} \stackrel{\tilde{\pi}}{\rightarrow} \widetilde{\Gamma_{g, 1}^{1}} \rightarrow 1 .
\end{aligned}
$$

Let $e \in H^{2}\left(\Gamma_{g}^{1} ; \mathbb{Z}\right)$ be the Euler class of the central extension

$$
1 \rightarrow \mathbb{Z} \rightarrow \Gamma_{g, 1} \rightarrow \Gamma_{g}^{1} \rightarrow 1
$$

The class $e$ may be regarded as a cohomology class in $H^{2}\left(\Gamma_{g, 1}^{1}, \Gamma_{g, 1} \times \mathbb{Z} ; \mathbb{Z}\right)$ in an obvious way. We define

$$
\begin{aligned}
& m_{i, j}:=\pi_{!}\left(e^{i} \omega^{j}\right) \in H^{2 i+j-2}\left(\Gamma_{g, 1} ; \bigwedge^{j} H\right) \\
& \widetilde{m_{i, j}}:=\widetilde{\pi}_{!}\left(e^{i} \omega^{j}\right) \in H^{2 i+2 j-2}\left(\widetilde{\Gamma_{g, 1}} ; \mathbb{Z}\right)
\end{aligned}
$$

for $i, j \in \mathbb{N}$. Here $\pi_{!}$and $\tilde{\pi}_{!}$are the fiber integrals introduced in the previous section. Clearly $m_{i+1,0}$ and $\widetilde{m_{i+1,0}}$ are equal to (the image of) the $i$-th Morita-Mumford (tautological) class $e_{i}\left(=\kappa_{i}\right) \in H^{2 i}\left(\Gamma_{g} ; \mathbb{Z}\right)[\mathrm{Mo}][\mathrm{Mu}]$ :

$$
m_{i+1,0}=\widetilde{m_{i+1,0}}=e_{i} \in H^{2 i}\left(\Gamma_{g, 1} ; \mathbb{Z}\right) .
$$

Remark 3.10. Let $\mathcal{F}_{g-1}$ be the dressed moduli of pairs of compact Riemann surfaces of genus $g$ and holomorphic line bundles of degree $g-1$ on the surfaces. The space $\mathcal{F}_{g-1}$ is aspherical and its $\pi_{1}$ is equal to $\widetilde{\Gamma_{g, 1}}$. As is known, the Lie algebra of holomorphic differential operators "near $S^{1}$ " has an infinitesimal and transitive action on the dressed moduli $\mathcal{F}_{g-1}$ [ADKP]. The $\widetilde{m_{i, j}}$ 's have their origins in the equivariant cohomology of $\mathcal{F}_{g-1}$ under this action [Ka1]. 


\section{Evaluations.}

The purpose of this section is to evaluate the classes $m_{2,0}, m_{1,1}$ and $m_{0,3}$ and to prove that $H^{r}\left(\Gamma_{g, 1} ; \bigwedge^{s} H_{1}\left(\Sigma_{g, 1} ; \mathbb{Q}\right)\right)$ is generated by $m_{i, j}$ 's for the case $r+s=2$ and the case $g \geq 5$ and $(r, s)=(1,3)$.

Denote by $\Omega$ the symplectic form on $H$ induced by the cup product:

$$
\Omega:=\sum_{i=1}^{g} a_{i} \otimes b_{i}-b_{i} \otimes a_{i} \in \bigwedge^{2} H,
$$

where $\left\{a_{i}, b_{i} ; 1 \leq i \leq g\right\}$ is (the homology classes induced by) a symplectic generator system of the fundamental group $\pi_{1}\left(\Sigma_{g, 1}\right)$ as in $\S 2$.

\section{Proposition 4.1.}

$$
m_{0,2}=\pi_{!}\left(\omega^{2}\right)=2 \Omega \in H^{0}\left(\Gamma_{g, 1} ; \bigwedge^{2} H\right)
$$

Proof. It suffices to show that

$$
<\omega^{2},\left[\Sigma_{g, 1}, \partial\right]>=2 \Omega
$$

Here $\left[\Sigma_{g, 1}, \partial\right]$ is a 2 -chain introduced in (2.2). Since $\omega\left(\widetilde{w_{4 i}}\right)=0$, we have

$$
\begin{aligned}
& <\omega^{2},\left[\Sigma_{g, 1}, \partial\right]>=\sum_{j=1}^{4 g} \omega^{2}\left(\widetilde{w_{j-1}}, w_{j}\right)-\sum_{i=1}^{g}\left(\omega^{2}\left(a_{i}, a_{i}^{-1}\right)+\omega^{2}\left(b_{i}, b_{i}^{-1}\right)\right) \\
= & \sum_{i=1}^{g} a_{i} \wedge b_{i}-\left(a_{i}+b_{i}\right) \wedge a_{i}-\left(a_{i}+b_{i}-a_{i}\right) \wedge b_{i}+a_{i} \wedge a_{i}+b_{i} \wedge b_{i} \\
= & \sum_{i=1}^{g} a_{i} \wedge b_{i}-b_{i} \wedge a_{i}=2 \Omega,
\end{aligned}
$$

as was to be shown.

Next we study the classes $m_{1,1}$ and $m_{0,3}$. In [Mo1] and [Mo2] Morita proved

$$
H^{1}\left(\Gamma_{g, 1} ; H\right)=\mathbb{Z}, \quad \text { and } \quad H^{1}\left(\Gamma_{g, 1} ; \bigwedge^{3} H\right)=\mathbb{Z}^{2}
$$

where we denote $H=H_{1}\left(\Sigma_{g, 1} ; \mathbb{Z}\right)$ as before. Our results are

Theorem 4.3. The class $m_{1,1}$ generates the group $H^{1}\left(\Gamma_{g, 1} ; H\right)$.

Theorem 4.4. If $g \geq 5$, the classes $m_{0,2} m_{1,1}$ and $m_{0,3}$ generate the group $H^{1}\left(\Gamma_{g, 1}\right.$; $\left.\wedge^{3} H \otimes \mathbb{Q}\right)$.

The rest of this section is devoted to the proof of the theorems. As was shown by Harer $[\mathrm{H}]$, if $g \geq 3$, we have $H^{2}\left(\Gamma_{g, 1} ; \mathbb{Q}\right)=\mathbb{Q}$ and the class $m_{2,0}=e_{1}$ generates it. Hence in the case $r+s=2$ the groups $H^{r}\left(\Gamma_{g, 1} ; \bigwedge^{s} H \otimes \mathbb{Q}\right)$ are generated by the classes $m_{i, j}$ 's. Consequently 
Corollary 4.5. If $g \geq 3$, the group $H^{2}\left(\widetilde{\Gamma_{g, 1}} ; \mathbb{Q}\right)$ is isomorphic to $\mathbb{Q}^{3}$ and the classes $\widetilde{m_{0,2}}, \widetilde{m_{1,1}}$ and $\widetilde{m_{2,0}}$ form its free basis. $\S 5)$

The first half of the corollary has been already shown by Arbarello et. al.([ADKP]

To prove the theorems we endow the surface $\Sigma_{g}$ with a Riemannian metric. Fix a sufficiently small positive real $\epsilon$. Let $\varpi: S T \Sigma_{g} \rightarrow \Sigma_{g}$ be the unit tangent bundle of the surface $\Sigma_{g}$. Denote by $D^{2}$ the unit disk in $\mathbb{C}: D^{2}:=\{z \in \mathbb{C} ;|z| \leq 1\}$. We define a disk bundle $D_{g}$ over $S T \Sigma_{g}$ by

$$
D_{g}:=\left\{\left(v_{1}, x_{2}\right) \in S T \Sigma_{g} \times \Sigma_{g} ; \operatorname{dist}\left(\varpi\left(v_{1}\right), x_{2}\right) \leq \epsilon\right\},
$$

The first projection induces its projection $p_{1}: D_{g} \rightarrow S T \Sigma_{g}$. The disk bundle is trivial through the projection

$$
S T \Sigma_{g} \times D^{2} \rightarrow D_{g}, \quad(v, z) \mapsto\left(v, \operatorname{Exp}_{\varpi(v)}(\epsilon z v)\right) .
$$

Here we use the (almost) complex structure induced by the given Riemannian metric.

Consider a $\Sigma_{g, 1}$-bundle

$$
p_{1}: Y_{g}\left(:=S T \Sigma_{g} \times \Sigma_{g}-\operatorname{int} D_{g}\right) \rightarrow S T \Sigma_{g}
$$

induced by the first projection. The fundamental group $\pi_{1}\left(S T \Sigma_{g}\right)$ is embedded into the group $\Gamma_{g, 1}$ through the classifying map $\iota$ of the bundle $p_{1}: Y_{g} \rightarrow S T \Sigma_{g}$, and is identified with the kernel of the forgetting map $\Gamma_{g, 1} \rightarrow \Gamma_{g}$ :

$$
1 \rightarrow \pi_{1}\left(S T \Sigma_{g}\right) \stackrel{\iota}{\rightarrow} \Gamma_{g, 1} \rightarrow \Gamma_{g} \rightarrow 1
$$

Since the spaces $\Sigma_{g}, S T \Sigma_{g}, D_{g}$ and $Y_{g}$ are all aspherical, we drop the notations $\pi_{1}(\cdot)$ in the cohomology groups.

The identity map $1_{H} \in \operatorname{Hom}(H, H)$ induces a cohomology class

$$
1_{H} \in H^{1}\left(\Sigma_{g} ; H\right) \cong \operatorname{Hom}(H, H) \text {. }
$$

By abuse of notation we denote also by $1_{H}$ the pull-back $\varpi^{*}\left(1_{H}\right)$ through the projection $\varpi: S T \Sigma_{g} \rightarrow \Sigma_{g}$ :

$$
1_{H}=\varpi^{*}\left(1_{H}\right) \in H^{1}\left(S T \Sigma_{g} ; H\right) \cong \operatorname{Hom}(H, H) .
$$

In [Mo1] Morita proved the following theorem (see also [Mo2] p.81 $1.4 \mathrm{ff}$ ).

Theorem 4.6 (Morita).

$$
H^{1}\left(\Gamma_{g, 1} ; H\right)=\mathbb{Z}
$$

Furthermore a crossed homomorphism $k: \Gamma_{g, 1} \rightarrow H$ represents a generator of the group $H^{1}\left(S T \Sigma_{g} ; H\right)$ if and only if the restriction of $k$ to $\pi_{1}\left(S T \Sigma_{g}\right)$ is equal to $\pm(2-2 g) 1_{H}$ :

$$
\iota^{*}(k)= \pm(2-2 g) 1_{H} \in H^{1}\left(S T \Sigma_{g} ; H\right) .
$$

As for $\bigwedge^{3} H=\wedge^{3} H_{1}\left(\Sigma_{g, 1} ; H\right)$ he proved the following ([Mo3] Theorem 5.1, see also the proof of Corollary 5.7). Let $k_{0}$ be a generator of the group $H^{1}\left(\Gamma_{g, 1} ; H\right)$. 
Theorem 4.7 (Morita). If $g \geq 3$,

$$
H^{1}\left(\Gamma_{g, 1} ; \bigwedge^{3} H\right)=\mathbb{Z} \oplus \mathbb{Z}
$$

The class $\Omega \wedge k_{0}$ and a class he named $2 \tilde{k}$ form its free basis. Furtheremore their restriction to $\pi_{1}\left(S T \Sigma_{g}\right)$ are given by

$$
\begin{aligned}
& \iota^{*}\left(\Omega \wedge k_{0}\right)= \pm(2-2 g) \Omega \wedge 1_{H} \in H^{1}\left(S T \Sigma_{g} ; \bigwedge^{3} H\right) \\
& \iota^{*}(2 \tilde{k})=2 \Omega \wedge 1_{H} \in H^{1}\left(S T \Sigma_{g} ; \bigwedge^{3} H\right) .
\end{aligned}
$$

Therefore our theorems are reduced to

Assertion 4.8.

$$
\begin{aligned}
& \iota^{*}\left(m_{1,1}\right)=-(2-2 g) 1_{H} \in H^{1}\left(S T \Sigma_{g} ; H\right) \\
& \iota^{*}\left(m_{0,3}\right)=-6 \Omega \wedge 1_{H} \in H^{1}\left(S T \Sigma_{g} ; \bigwedge^{3} H\right)
\end{aligned}
$$

In fact, (1) implies Theorem 4.3 by Theorem 4.6. So we have $m_{2,0} m_{1,1}= \pm 2 \Omega \wedge$ $k_{0}$. From Theorem 4.7 the class $m_{0,3}$ has a representation $m_{0,3}=a \Omega \wedge k_{0}+b(2 \tilde{k})$ for some integers $a$ and $b$. Since $H^{1}\left(S T \Sigma_{g} ; \bigwedge^{3} H\right)=H \otimes \bigwedge^{3} H$ is $\mathbb{Z}$-free, we have

$$
-6= \pm a(2-2 g)+2 b
$$

and so $b \equiv-3 \bmod (g-1)$, while $g-1 \geq 4$. Thus we have $b \neq 0$.

This completes the proof of Theorems 4.3 and 4.4 modulo Assertion 4.8.

Let $M$ be a $\pi_{1}\left(S T \Sigma_{g}\right)$-module. By excision we may consider the map

$$
j^{*}: H^{*}\left(Y_{g}, \partial Y_{g} ; M\right) \underset{\text { exc. }}{\cong} H^{*}\left(S T \Sigma_{g} \times \Sigma_{g}, D_{g} ; M\right) \rightarrow H^{*}\left(S T \Sigma_{g} \times \Sigma_{g} ; M\right)
$$

The fiber integral $p_{1 !}: H^{*}\left(Y_{g}, \partial Y_{g} ; M\right) \rightarrow H^{*-2}\left(S T \Sigma_{g} ; M\right)$ decomposes itself into

$$
H^{*}\left(Y_{g}, \partial Y_{g} ; M\right) \stackrel{j^{*}}{\rightarrow} H^{*}\left(S T \Sigma_{g} \times \Sigma_{g}, D_{g} ; M\right) \stackrel{p_{1}}{\rightarrow} H^{*-2}\left(S T \Sigma_{g} ; M\right)
$$

Here the latter fiber integral $p_{1 !}$ is the usual one induced by the first projecion $p_{1}: S T \Sigma_{g} \times \Sigma_{g} \rightarrow S T \Sigma_{g}$. Thus we have

$$
\iota^{*} m_{1,1}=p_{1 !} j^{*}(e \omega) \text { and } \iota^{*} m_{0,3}=p_{1 !} j^{*}\left(\omega^{3}\right) .
$$

Now we have

$$
\begin{aligned}
& j^{*}(e)=p_{2}{ }^{*} e^{\prime} \in H^{2}\left(S T \Sigma_{g} \times \Sigma_{g} ; \mathbb{Z}\right) \\
& j^{*}(\omega)=p_{2}{ }^{*} 1_{H}-p_{1}{ }^{*} 1_{H} \in H^{1}\left(S T \Sigma_{g} \times \Sigma_{g} ; H\right),
\end{aligned}
$$

where $p_{2}: S T \Sigma_{g} \times \Sigma_{g} \rightarrow \Sigma_{g}$ is the second projection and

$$
e^{\prime}=e\left(T \Sigma_{g}\right) \in H^{2}\left(\Sigma_{g} ; \mathbb{Z}\right) .
$$


Since $e^{\prime} 1_{H} \in H^{3}\left(\Sigma_{g} ; H\right)=0$, we have

$$
\begin{aligned}
\iota^{*} m_{1,1}=p_{1 !} j^{*}(e \omega)=p_{1 !}\left(p_{2}{ }^{*} e^{\prime}\right)\left(p_{2}{ }^{*} 1_{H}-p_{1}{ }^{*} 1_{H}\right) \\
\quad=-\left(p_{1 !} p_{2}{ }^{*} e^{\prime}\right) 1_{H}=-(2-2 g) 1_{H} .
\end{aligned}
$$

On the other hand, since $\left(1_{H}\right)^{3} \in H^{3}\left(\Sigma_{g} ; \bigwedge^{3} H\right)=0$ and $p_{1 !} p_{2}{ }^{*} 1_{H} \in H^{-1}\left(S T \Sigma_{g}\right.$; $H)=0$, we have

$$
j^{*}\left(\omega^{3}\right)=\left(p_{2}{ }^{*} 1_{H}-p_{1}{ }^{*} 1_{H}\right)^{3}=-3\left(p_{2}{ }^{*}\left(1_{H}\right)^{2}\right) p_{1}{ }^{*} 1_{H}+3\left(p_{2}{ }^{*} 1_{H}\right) p_{1}{ }^{*}\left(1_{H}\right)^{2}
$$

and

$$
p_{1 !} j^{*}\left(\omega^{3}\right)=-3\left(p_{1 !} p_{2}{ }^{*}\left(1_{H}\right)^{2}\right) 1_{H}+3\left(p_{1 !} p_{2}{ }^{*} 1_{H}\right)\left(1_{H}\right)^{2}=-3<\left(1_{H}\right)^{2},\left[\Sigma_{g}\right]>1_{H},
$$

where we denote by $\left[\Sigma_{g}\right] \in H_{2}\left(\Sigma_{g} ; \mathbb{Z}\right)$ the fundamental class. From a similar calculation to Proposition 4.1 follows $\left\langle\left(1_{H}\right)^{2},\left[\Sigma_{g}\right]\right\rangle=2 \Omega$. Therefore

$$
\iota^{*} m_{0,3}=p_{1 !} j^{*}\left(\omega^{*}\right)=-6 \Omega \wedge 1_{H}
$$

This completes the proof of Assertion 4.8 and so those of Theorems 4.3 and 4.4.

Remark 4.9. The crossed homomorphism $\tilde{k}=\frac{1}{2} 2 \tilde{k}: \Gamma_{g, 1} \rightarrow \frac{1}{2} \bigwedge^{3} H$ in (4.7) is the Johnson homomorphism extended to the whole mapping class group by Morita [Mo3]. Hence Theorem 4.4 implies the Johnson homomorphism $\tilde{k}$ is represented by $m_{0,3}$ and $m_{0,2} m_{1,1}$ over $\mathbb{Q}$. The author, however, doesn't know the explicit representation of $\tilde{k}$ by $m_{0,3}$ and $m_{0,2} m_{1,1}$.

\section{REFERENCES}

[ADKP] E. Arbarello, C. DeContini, V.G. Kac, and C. Procesi, Moduli spaces of curves and representation theory, Commun. Math. Phys. 117 (1988), 136.

[H] J.L. Harer, The second homology group of the mapping class group of an orientable surface, Invent. math. 72 (1983), 221-239.

[HS] G. Hochshild and J.-P. Serre, Cohomology of group extensions, Trans. Amer. Math. Soc. 74 (1953), 110-134.

[I] A. Ishida, Master Thesis, (in Japanese), Univ. of Tokyo (1994).

[Ka] N. Kawazumi, Homology of hyperelliptic mapping class groups for surfaces, preprint. Hokkaido Univ. 262.

[Ka1] Moduli space and complex analytic Gel'fand-Fuks cohomology of Riemann surfaces, III, in preparation.

[Me] W. Meyer, Die Signatur von Flächenbündeln, Math. Ann. 201 (1973), 239-264.

[Mo] S. Morita, Characteristic classes of surface bundles, Inventiones math. 90 (1987), 551-577.

[Mo1] , Families of Jacobian manifolds and characteristic classes of surface bundles, I, Ann. Inst. Fourier 39 (1989), 777-810.

[Mo2] , Families of Jacobian manifolds and characteristic classes of surface bundles, II, Math. Proc. Camb. Phil. Soc. 105 (1989), 79-101.

[Mo3] , The extension of Johnson's homomorphism from the Torelli group to the mapping class group, Invent. math. 111 (1993), 197-224.

[Mu] D. Mumford, Towards an enumerative geometry of the moduli space of curves, Arithmetic and Geometry., Progr. Math. 36 (1983), 271-328. 